\title{
Enfoques intraindividual e interindividual en programas de pensamiento crítico
}

\author{
Carlos Javier Ossa Cornejo \\ Universidad del Bio-Bio - Chillán - Chile \\ Alejandro Díaz Mujica \\ Universidad de Concepción - Concepción - Chile
}

\begin{abstract}
Resumen
En la actualidad, hay diversas conceptualizaciones acerca del pensamiento crítico, clasificándolo mayoritariamente en definiciones, perspectivas metodológicas e instrumentos. Este artículo de revisión teórica tiene por objetivo realizar una clasificación de orden ontológico, analizando las finalidades que definen al pensamiento crítico como praxis humana; proponiéndose dos enfoques, uno como proceso intrapersonal, considerándolo un proceso de filtro interno de información; otro, como proceso interpersonal, donde el pensamiento es de naturaleza interactiva y culturalmente situada. Se realiza además una revisión bibliográfica, analizando programas aplicados en educación superior, relacionando programas y estudios en ambos enfoques, en base a objetivos y procedimientos de trabajo. Se concluye que el pensamiento crítico es un proceso complejo, que ha experimentado una evolución conceptual y epistemológica que lo orienta actualmente hacia una praxis social más que meramente individual, puesto que se encuadra en contextos y acciones que promueven la interacción entre personas.
\end{abstract}

Palabras clave: Pensamiento crítico; cognición; interacción social.

\section{Intraindividual and interindividual approaches in critical thinking programs}

\begin{abstract}
Currently, there are several conceptualizations about critical thinking, most of them classify it in definitions, methodological approaches and instruments. Objective of this article is to offer an ontological classification based on theoretical review, analyzing goals define critical thinking as human praxis, considering two approaches: intrapersonal process, seeing it as a process of refining information; and as interpersonal process where thought is interactive in its nature and culturally situated. A literature review was carried out, analyzing programs developed in higher education, and linking those programs to approaches, based on objectives and working procedures. We conclude that critical thinking is a complex process, which has experienced a conceptual and epistemological evolution, guiding it today towards social praxis and not merely individual, because of it's framing in epistemology and actions that promote interaction among people.
\end{abstract}

Keywords: Critical thinking; cognition; social interaction.

\section{Enfoques interindividual e interindividual em programas de pensamento crítico}

\section{Resumo}

Na atualidade, há diversas conceitualizações sobre o pensamento crítico, classificando-o principalmente em definições, perspectivas metodológicas e instrumentos. Este artigo de revisão teórica tem por objetivo realizar uma classificação de ordem ontológica, analisando as finalidades que definem o pensamento crítico como práxis humana, propondo-se dois enfoques, um como processo intrapessoal, considerando-o um processo de filtro interno de informação; outro, como processo interpessoal, em que o pensamento é de natureza interativa e culturalmente situada. Realiza-se, também, uma revisão bibliográfica, analisando programas aplicados na educação superior, relacionando programas e estudos em ambos os enfoques, na base de objetivos e procedimentos de trabalho. Conclui-se que o pensamento crítico é um processo complexo, que experimentou uma evolução conceptual e epistemológica que o orienta atualmente para uma práxis social mais que meramente individual, posto que se enquadra em contextos e ações que promovem a interação entre pessoas.

Palavras-chave: Pensamento crítico; cognição; interação social. 


\section{Introducción}

El pensamiento crítico es un proceso cognitivo que ha ido generando un amplio interés en áreas de las ciencias sociales y humanidades, así como en aquellas relacionadas con la formación profesional (Hawe, 2003). Es un concepto de difícil definición, puesto que puede entenderse desde diversas perspectivas (Davies, 2013; López, 2012; Paul \& Elder 2003).

Existen algunas propuestas de sistematización de definiciones y bases epistemológicas que sustentan al pensamiento crítico, y si bien son descriptivas, permiten conocer y evaluar tanto conceptos como habilidades que los diferentes autores han relevado para cualificarlo, medirlo y analizar los diferentes instrumentos de medición que se han utilizado (Olivares \& Heredia, 2012; Saiz \& Rivas, 2011; Yang, 2012). Aun cuando se han planteado diversos análisis respecto a las orientaciones que ha tomado el concepto (Akdere, 2012; Davies, 2013; Saiz \& Rivas, 2011), así como ciertas tipologías sobre el nivel de logro que puede alcanzarse (Paul \& Elder, 2003), no se han encontrado trabajos que permitan saber a cuál perspectiva epistémico-conceptual desde la tradición psicológica están relacionadas con aquel; tampoco se han encontrado estudios, que permitan comprender los sentidos y alcances de la praxis que se desea lograr al implementar este complejo proceso.

Esto es relevante debido a que muchos programas y experiencias de aplicación que han utilizado el pensamiento crítico, han focalizado habilidades e instrumentos sin detenerse a considerar su naturaleza y la finalidad con la que se exprese, lo cual se analiza desde la ontología (Guba \& Lincoln, 2002). Lo anterior genera, por un lado, que exista una diversidad de instrumentos que miden el constructo, enfocándose en habilidades cognitivas que se consideran, en función de la tradición psicológica que se plantee de la tradición cognitiva, bien individuales o bien sociales. Existen perspectivas ontológicas en la teoría cognitiva, respecto al ser humano y la mente, muy diferentes entre sí, e incluso hasta contrarias.

Esta dificultad respecto al análisis de las diferentes tradiciones ontológicas, y en las que poco se ha profundizado (Davies, 2013), no ha permitido tener un eje de análisis claro que permita comprender si las dificultades y potencialidades de estos programas, lo que se convierte en un obstáculo para establecer un consenso respecto a cómo se entiende y se promueve el pensamiento crítico (Abrami \& cols., 2008; Bensley \& Spero, 2014; Davies, 2013).

Se propone de esta manera un análisis ontológico en base a la naturaleza psicológica del pensamiento crítico como proceso cognitivo, y en la finalidad que alcanza como praxis social, analizando además algunos programas de promoción de pensamiento crítico en función de la organización propuesta.

\section{Conceptualización de pensamiento crítico}

El pensamiento crítico se ha definido como un tipo de pensamiento elaborado, es decir, como un proceso cognitivo que implica evaluación y reflexión (Daniel \& Auriac; 2014;
Eterovich \& Stiepovic, 2010), permite la construcción de un conocimiento nuevo, y la utilización estratégica del mismo en la vida cotidiana (Butler, 2012; Marin \& Halpern, 2011). Es definido también como un tipo de proceso cognitivo complejo, integrado por subprocesos interrelacionados que permiten evaluar, procesar analítica y reflexivamente, enjuiciar y aceptar o rechazar, información producida en contextos sociales o en trabajos científicos (Harada, 2009).

Es un modo de pensar en el cual el sujeto mejora la calidad de dicho proceso al apoderarse de las estructuras inherentes del acto de pensamiento y al someterlas a estándares intelectuales (Paul \& Elder, 2003). Se considera además como una habilidad de pensamiento que permite evaluar el mérito, la precisión, y/o autenticidad de lo que se está aprendiendo o elaborando, por lo que resulta una habilidad importante para el desarrollo de profesionales científicos (Harada, 2009). Se le conceptualiza así mismo como un mecanismo cognitivo que filtra las intenciones ideológicas mediante el continuo cuestionamiento de las prácticas de producción de conocimiento, y el reconocimiento de sus diferentes perspectivas (Montero, 2010).

Es una actividad reflexiva, en el sentido de que permite analizar el nivel de fundamento de una información o idea, en base tanto a la propia reflexión como a la reflexión de otros (López, 2012; Yang, 2012). Es además un proceso auto-dirigido, auto-disciplinado, autorregulado y auto-corregido, que propone someterse a rigurosos estándares de excelencia y dominio consciente de su uso; implicando una comunicación efectiva y el desarrollo de habilidades de resolución de problemas (Marin \& Halpern, 2011). Además se entiende por pensamiento crítico el comportamiento que se interesa y cuestiona los fundamentos en los que se asientan las ideas, acciones y juicios, tanto propios como ajenos (Oyler \& Romanelli, 2014).

De este modo, a partir de las diversas definiciones, se puede sintetizar que el pensamiento crítico es un proceso cognitivo de análisis y juicio que permite buscar las razones que fundamentan la información escrita u oral para evaluar su validez e intencionalidad.

\section{Proceso complejo y dinámico}

Se puede considerar al pensamiento crítico como un proceso de mejoramiento del pensamiento, orientado a que la persona sea más efectiva al utilizarlo en su medio sociocultural, y que tiene dos grandes dimensiones: por un lado, la posibilidad de desarrollar habilidades cognitivas y metacognitivas, tales como análisis, evaluación e inferencia; por otro lado, la posibilidad de generar autorregulación y motivación con el fin de lograr una disposición positiva hacia esos procesos, lo que implica estar abierto a enfoques múltiples, tomar decisiones e intervenir en la realidad social (Dwyer, Hogan, \& Stewart, 2014; Saiz \& Rivas, 2008).

De este modo, si bien es un proceso básicamente cognitivo, se ve complementado por procesos motivacionales que le permiten mantener el esfuerzo que requiere este conjunto de procesos (Abrami \& cols., 2008; Valenzuela, 
Nieto, \& Muñoz, 2014). Es posible, en este sentido, caracterizar al pensamiento crítico como proceso de conflicto cognitivo, al considerarlo como habilidad que permite cuestionar y evaluar, lo que supone problematizar el conocimiento que se tiene con la finalidad de mejorarlo y tomar decisiones adecuadas (Bouzas, 2009; Olivares \& Heredia, 2012); esto se ha relacionado con el aprendizaje basado en problemas, permitiendo además el fortalecer el trabajo cooperativo. El desarrollo de actividades de resolución de problemas es un método que permite el fomento de pensamiento crítico en estudiantes universitarios (Sánchez, 2012; Tiwari, Lai, So, \& Yuen, 2006), esto dado que implicaría asumir un esfuerzo de cambio de esquemas cognitivos, para establecer conexiones y evaluar los pasos realizados, así como para generar conclusiones razonadas y reflexivas.

Estos componentes caracterizarían al pensamiento crítico como un proceso complejo, que incluiría habilidades cognitivas, metacognición, disposición al juicio crítico, y colaboración frente a resolución de problemas (Olivares, Saiz, \& Rivas, 2013; Saiz \& Rivas, 2011; Yang, 2012; Tiwari \& cols, 2006).

\section{Enfoques intraindividual e interindividual como ejes de la tradición cognitiva para el análisis del pensamiento crítico}

Se ha generado una larga discusión respecto a si el pensamiento crítico es una habilidad general del ser humano como faceta del pensamiento, o por el contrario, es una habilidad relacionada a un tema o contenido en específico, ya que el pensamiento requiere de un elemento para expresarse (Abrami \& cols., 2008; Bensley \& Spero, 2014). Aun cuando no hay acuerdo respecto a estas dos miradas, se puede reflexionar respecto a esta discusión sobre la necesidad de situar a esta habilidad dentro de una finalidad práctica que le otorgue una posibilidad de uso por parte de las personas, así como su manipulación y evaluación (Davies, 2013).

Proponemos que existe otra vía para dotar al pensamiento crítico de un sentido de praxis social, la que estaría relacionada con perspectivas cognitivas diferentes, una de ellas, la tradicional cognitiva-conductual, y la otra, constructivista. Estas dos perspectivas de la teoría cognitiva han entendido los procesos cognitivos como aspectos diferentes de la praxis humana, por un lado, como conjunto de procesos individuales y reducidos a procesos unitarios; por otro lado, como un conjunto de procesos que permiten la contextualización y estimulación de la cognición a través de interacciones socioculturales (Hernández, 2007).

Podría entenderse entonces el pensamiento crítico, como un proceso orientado mayoritariamente hacia el razonamiento, es decir, que puede regirse y analizarse según las reglas de la lógica, o como proceso mental que puede mejorarse y fortalecerse como aspecto intelectual, considerándolo un proceso mental de cognición individual (intraindividual), centrándose en cómo la persona utiliza su cognición para filtrar información (Akdere, 2012; Hawe, 2003), relacionándose con la perspectiva psicológica cognitiva (tanto la tradi- cional como la de construcción individual piagetana), centrada en utilizar el pensamiento para sistematizar o transformar la información en base al pensamiento del propio individuo (Beas, Santa Cruz, Thomsen, \& Utreras, 2003).

Por otro lado, se observa que otra finalidad del pensamiento crítico se orienta al cambio de comportamientos o situaciones sociales o al desarrollo de productos culturales, del ámbito de las humanidades y la educación, siendo ambos tipos coincidentes en ver esta habilidad como un proceso mental que parte del individuo, pero orientado a lo social (interindividual) ya que se basa en como el proceso cognitivo se enmarca en procesos comunicativos y relacionales para generar un producto cultural (Akdere, 2012; Hawe, 2003; Montero, 2010). Este enfoque podría relacionarse con la perspectiva psicológica socioconstructivista, pues considera esta habilidad como un proceso de interacción, enfocado a contextualizar el conocimiento según marcos culturales y sociales (Kozulin, 2006) y entendiendo la cognición como proceso distribuido entre varios actores (Hernández, 2007).

\section{Programas de pensamiento crítico desde el enfoque intraindividual}

Las miradas que centran el pensamiento crítico en este enfoque, priorizan el uso de esta habilidad como proceso cognitivo concreto y aplicable, en relación con habilidades que permiten el logro de un pensamiento productivo (Nickerson, Perkins, \& Smith, 1998), es decir, que puede mejorarse y de ese modo dar una utilidad al intelecto. Se relevan así, procesos como la interpretación, el análisis, la evaluación, la inferencia, la explicación y la autocorrección (Olivares \& Heredia, 2012). Se puede observar que el pensamiento crítico se considera una habilidad conformada por una serie de componentes específicos, habitualmente descritos como factores cognitivos que operan concurrentemente para desarrollar la habilidad, es decir, que se conjugan para tomar la información que se analiza, se evalúa, se interpreta y se dispone para entregar una respuesta frente a situaciones del medio (Olivares \& Heredia, 2012).

Dicha perspectiva considera habilidades básicas (o focalizadas) de análisis, pensamiento (inductivo-deductivo) interpretación, evaluación, y argumentación, las cuales se trabajan como elementos individuales, pero se traducen en un proceso complementario, permitiendo la expresión de la habilidad de pensamiento crítico. Esta aproximación permite la identificación y medición de estos elementos de modo objetivo, ya que los instrumentos construidos se centran en situaciones que permiten reconocer estos procesos.

Uno de los programas que se han desarrollado en esta línea es el de Filosofía para niños de Lipman, que se centra en el desarrollo de habilidades para cuestionar lo que los niños y niñas aprenden y manejan como información (Nickerson \& cols., 1998). Su objetivo es hacer accesible el proceso de investigación filosófica para niños y niñas puesto que, por un lado, se valora el reconocimiento de los derechos de los niños y jóvenes como pensadores; por otro lado, 
la filosofía cumple una función central en el desarrollo integral del ser humano, y no como disciplina necesariamente, sino como forma de vida (Carmona, 2005).

En una línea similar se encuentra el desarrollo de habilidades metacognitivas, que se orienta al desarrollo de estrategias que permitan analizar las decisiones que deben tomar los estudiantes frente a sus actividades de aprendizaje, o a la resolución de un problema, lo que permitiría que el alumno dirija su pensamiento para supervisar y controlar lo que piensa y hace (Mateos, 2001). Otros programas en esta misma perspectiva son el método de pensamiento verbalizado (Think Aloud) en donde los estudiantes deben decir en voz alta (verbalización), aquellas ideas y razones que surgen del proceso de aprendizaje, y muestran los procesos cognitivos evocados en la tarea que realizan, lo que les permite hacer conscientes sus procesos cognitivos y motivacionales (Ku \& Ho, 2010).

Finalmente, se encuentran los programas de enseñanza de pensamiento formal, en los que se desarrollan habilidades de pensamiento abstracto a través de temas o materias que guardan relación con el proceso formativo institucional mediante un proceso de exploración, invención y aplicación de los contenidos vistos en el proceso de enseñanza y aprendizaje. Entre éstos se puede considerar el modelo ADAPT (Accent on the Development of Abstract Process of Thought), dirigido a diversas áreas de formación, y centrado en la planificación de los contenidos para estimular el pensamiento formal en los estudiantes; el modelo DOORS (Development Of Operational Reasoning Skills), que busca de igual modo trabajar el pensamiento formal en los estudiantes universitarios; pero se diferencia en que está más orientado a las necesidades del curriculum tradicional y con materias convencionales; el COMPAS (Consortium for Operating and Managing Programs for the Advancement of Skills), que se derivó del programa DOORS y se trabajó con siete programas de College en Estados Unidos entre otros (Nickerson \& cols., 1998).

Otros programas en esta línea, son el llamado ADDIE desarrollado por McGriff, y el de Dick y Carey (en Yang, 2012); el primero se compone de un conjunto de actividades organizadas en un formato instruccional sistemático que consiste en cinco fases: (1) Análisis, (2) Diseño, (3) Desarrollo, (4) Implementación, y (5) Evaluación. Por otra parte, el modelo de Dick y Carey plantea un modelo instruccional dividido en pequeñas partes, las que se organizan para, en conjunto, lograr el desarrollo de la habilidad; es un modelo integrativo, que toma aspectos cognitivos, personales y conductuales (Yang, 2012).

\section{Programas de pensamiento crítico desde el enfoque interindividual}

El enfoque centrado en el pensamiento crítico como aspecto orientado a las conductas sociales y al desarrollo de la cultura (enfoque interindividual), se podría relacionar con experiencias que buscan el logro de habilidades más complejas y holistas, como el enjuiciamiento, la comprobación de hipótesis, la toma de decisiones y la solución de problemas (Beltrán \& Torres, 2009). Dichas habilidades se desarrollan en un espacio sociocultural, y se enfocan en el planteamiento de los componentes como conjuntos de procesos integrados que permiten el desarrollo de análisis holístico, relacionándolo con un contexto y situados culturalmente, donde las habilidades específicas son subprocesos que reportan información a estos procesos más globales (Sánchez, 2012; Saiz \& Rivas, 2011); alineando por ello a esta mirada con la perspectiva socioconstructivista (Kozulin, 2006; Hernández, 2007).

Este enfoque tendría como foco dotar a la persona de una herramienta efectiva de adaptación a sus necesidades cotidianas y en función de su cultura. Además, se orientaría de mejor modo al desarrollo de tareas relacionadas con las actividades didácticas y curriculares en la formación universitaria sugeridas en la actualidad, especialmente las centradas en el desarrollo de estrategias decisionales en la formación de directivos, o en las propuestas de enseñanza de las ciencias, y en propuestas de formación comunitaria frente a la política y a los medios de información (Sotolongo \& Delgado, 2006; Tejada, 2000).

Se observa por otro lado, una perspectiva de mayor base social o centrada en la interacción, que plantea que el pensamiento crítico es un proceso cognitivo situado y definido por la comunicación, por lo que si bien, existe un procesamiento cognitivo, éste es complementado con recursos argumentativos que conlleva a una conducta para intervenir en el medio y en el grupo social (Saiz \& Rivas, 2011). De esta forma el pensamiento crítico es un elemento útil para comportarse de una manera efectiva en el medio social y generar cambios mediante la comunicación (interacción).

Estos programas comprenden el proceso de pensamiento crítico desde una estructura multidimensional, donde se encuentran de forma complementaria, los procesos cognitivos, metacognitivos, motivacionales y comunicacionales (Marinkovic, 2007; Valenzuela \& cols., 2014). Plantean que una manera de desarrollar aprendizajes significativos a través del pensamiento crítico, se da en base a situaciones que lleven a problematizar al estudiante y le motiven a plantear sus propias soluciones, las que deben ser argumentadas y reflexionadas, lo que establecería una estrecha relación entre el pensamiento crítico, el Aprendizaje Basado en Problemas (ABP) y la argumentación (Antequera, 2011; Olivares \& Heredia, 2012; Olivares \& cols., 2013).

Una de las experiencias que se ha utilizado es el programa CoRT (Cognitive Research Trust, traducido como Investigación Cognitiva Confiable), desarrollado por De Bono (2009), se basa en la distinción entre pensamiento lateral, el que es impredecible, no limitado por convenciones, ni necesariamente secuencial; y el pensamiento vertical, de características opuestas al anterior. Los contenidos se organizan en torno a una serie de preguntas que uno puede hacerse a sí mismo y pretenden fortalecer habilidades a través del desarrollo de estrategias que permiten identificar y considerar los factores que inciden en las ideas, priorizar los 
más relevantes, definir reglas para analizarlos, usar técnicas de predicción evaluación, determinar objetivos y propósitos y explorar otros puntos de vista que permitan redefinir la situación (De Bono, 2009).

Otra experiencia en el desarrollo de pensamiento crítico sustentado en la resolución de problemas es la llamada modelo SPRI, herramienta que permite desarrollar un conocimiento gracias a la construcción de información fundamentada, implicando que el estudiante tenga conocimientos previos del tema a tratar, sin embargo, facilita en el estudiante el rigor intelectual y la organización de sus conocimientos (Parra \& Lago de Vergara, 2003). Su estructuración se basa en describir los hechos o situaciones que sean más relevantes (Situación); identificar los problemas (Problema); buscar soluciones y alternativas a los problemas identificados, generando respuestas desde el punto de vista del estudiante (Resolución); y, consultar a otros autores o fuentes de información, u otros puntos de vista (Información) escritas u orales.

Un tercer programa es ASARPIC, que deriva su nombre de Aprendizaje Significativo a través de la Resolución de un Problema Integrador y Contextualizado (Sánchez, 2012), enfocado en el logro de un aprendizaje significativo a través de la resolución de un problema integrador y contextualizado. Este es un modelo para la enseñanza de las habilidades del pensamiento que permite su transferencia a través de los dominios del conocimiento, los que consideran cuatro componentes: el actitudinal o de disposición; la instrucción y la práctica de las habilidades del pensamiento crítico; actividades de transferencia a través de los contextos y el componente metacognitivo usado para dirigir y evaluar el pensamiento (Sánchez, 2012).

Otro programa que ha seguido esta perspectiva es el llamado ARDESOS (Saiz \& Rivas, 2011) cuyo nombre deriva de argumentación, decisión y resolución de problemas en la vida diaria, que serían los procesos subyacentes del modelo (Argumentation, Decision, Solving problems in daily Situations). Este se enfoca en enseñar las habilidades que se consideran esenciales para el desarrollo del pensamiento crítico y su utilización en actividades cotidianas de la persona (Saiz \& Rivas, 2011). Para lograr esto, se hace necesario usar el razonamiento, así como buenas estrategias de resolución de problemas, con el fin de generar una adecuada toma de decisiones, trabajando en base a situaciones problemas que deben analizarse en grupos de estudiantes para llegar a una decisión fundamentada.

Finalmente, y dando un leve giro en este enfoque, están aquellos programas donde se trabaja el pensamiento crítico desde la argumentación y el debate, basándose en una postura más cercana a la pragma-dialéctica, que apunta a identificar los movimientos dialógicos que se realizan en una argumentación; se ve al razonamiento relacionado con la argumentación desde un punto de vista interactivo, centrándose en un proceso de discurso argumentativo señalado como discusión crítica (Marinkovic, 2007).

Esta relación entre argumentación y pensamiento crítico descansa en la idea de que los argumentos son afirma- ciones que se plantean de un modo ordenado y persuasivo, donde algunas de ellas sirven de razones para otras, que conforman una conclusión, organizándolas de un modo en que resulten creíbles para otros (Govier, 2010; Saiz \& Rivas, 2008). De este modo, el proceso argumentativo puede orientarse, por un lado, como proceso cognitivo (razonamiento), o por otro lado, como proceso de comunicación social.

Puede señalarse un programa realizado en Argentina, entre 1998 y 2000 consistente en dos proyectos en los que postulaba la necesidad de que el aprendizaje de la argumentación oral (en alumnos que ingresan a la universidad), se refuerza con la práctica de estrategias cognitivas y metacognitivas que permiten el pensamiento crítico (Zamudio, Rolando, \& Ascione, 2002). El pensamiento crítico no se consideró solo como un proceso de análisis de información, sino como proceso metadiscursivo que permite confrontar un sistema de ideas y de valores presentados por otro, donde la adquisición de la competencia argumentativa ha permitido comprobar la presencia de diversas variables, tales como la práctica de la discusión, la interacción con un oponente y los saberes previos, y no solamente el manejo de estrategias exclusivamente retóricas (Zamudio \& cols., 2002).

Un segundo programa trabajado con estudiantes de pedagogía españoles buscó analizar los cambios que se producían en el pensamiento crítico de los participantes a través de un entorno virtual de aprendizaje(Martínez \& Pascual, 2013). Se reconoce un gran impacto de la intervención en el mayor desarrollo de una postura analítica y reflexiva en el intercambio de información, gracias a las actividades de interacción como el foro o los debates, lo que posibilita el desarrollo de profesionales reflexivos y abiertos a generar cambios en sus entornos.

En otro programa, desarrollado con estudiantes universitarios venezolanos, se trabajó una metodología de análisis argumentativo basada en dos etapas, la primera orientada a enseñarles a analizar argumentos a partir de textos presentados por el docente, y una segunda etapa enfocada en que los estudiantes escribieran un texto argumentativo en formato de ensayo donde incorporaran diversas perspectivas que apoyaran el tema seleccionado. Se observó que el uso de la información ofrecida por diferentes tipos de temas controversiales en una clase de lectura y escritura puede contribuir de manera significativa con la promoción y desarrollo del pensamiento crítico, la reflexión, el análisis y la argumentación de los estudiantes, tanto en forma oral como escrita (Mota de Cabrera, 2010).

\section{Consideraciones finales}

Respecto a su definición, podemos señalar que aun cuando existe diversidad de definiciones y miradas sobre el pensamiento crítico, se puede señalar que su esencia es eminentemente cognitiva, pues las definiciones apuntan a que es un proceso ideacional que analiza y evalúa de un modo sistemático y fundamentado la información derivada del medio, sea escrita, conductual o discursiva, que las personas 
encuentran en su vida diaria o en su desempeño laboral (Marin \& Halpern, 2011; Yang, 2012). Al pensar críticamente se buscan y analizan las razones que sustentan una afirmación o tesis, además es posible reconocer procesos de evaluación o juicio, al revisar las cualidades de validez de las razones encontradas; y de metacognición, al reflexionar de modo constante respecto a la cantidad y calidad de las razones y su validez (Beltrán \& Torres, 2009; Saiz \& Rivas, 2011).

Por otra parte, como habilidad es compleja y de alto nivel, pues reúne un conjunto de acciones cognitivas que permiten su desarrollo, como el razonamiento, el juicio y la metacognición, además de acciones motivacionales y de autorregulación que ayudan a mantener el esfuerzo cognitivo en el proceso de pensamiento crítico. $Y$ aun mas, se reconocen procesos discursivos y de interacción que potencian la capacidad cuestionadora y evaluativa (Marinkovic, 2007; Saiz \& Rivas, 2011; Valenzuela \& cols., 2014); permitiéndole una característica dinámica y autogeneradora que se observa por ejemplo en el desarrollo de debates argumentativos.

En tercer lugar, como praxis humana, el desarrollo del pensamiento crítico es un elemento fundamental para la vida de las personas, tanto en la vida cotidiana (Butler, 2012; Marin \& Halpern, 2011; Saiz\& Rivas, 2008), como en ámbitos profesionales (Eterovic \& Stiepovich, 2010; Martínez \& Pascual, 2013), ya que no solo está relacionada al desarrollo de una disciplina, sino que además, es un proceso relevante para los distintos estudiantes universitarios como parte de las competencias profesionales básicas que demanda el siglo XXI (Hawe, 2003), pues permite fortalecer el proceso de toma de decisiones (Saiz \& Rivas, 2011).

Finalmente, en relación con la propuesta de enfoques como ejercicio de análisis ontológico del pensamiento crítico, desde su finalidad como praxis humana, es necesario reconocer las diferentes perspectivas de la tradición cognitiva que han sustentado sus definiciones, instrumentos y programas para dotar de una mirada más comprensiva e integral dicho constructo, evitando los reduccionismos que le han caracterizado y han obstaculizado generar una propuesta consensuada. Proponemos este ejercicio como base para entenderlo como un proceso dialéctico, por un lado, se puede orientar al desarrollo de una acción individual y de construcción de conocimiento en un campo determinado; por otro lado, como una acción interindividual, sustentada en la interacción social y permitiendo el cambio social. Es posible ver que existe una interdependencia de la representación cognitiva individual y social (Marková, 2006), puesto que en una dimensión se observa como factor intrapersonal, de reflexión y manejo del conocimiento y cercano a la concepción cognitiva tradicional, mientras que en otra (proceso interpersonal), está orientado a analizar y evaluar el contexto sociocultural para generar cambios en la realidad cotidiana y más cercano a la postura socioconstructivista.

Así mismo se observan muchos programas que pueden organizarse en estos dos enfoques propuestos; aquellos que promueven y buscan mayoritariamente el desarrollo cognitivo y el procesamiento reflexivo de la información como los programas de mejoramiento del pensamiento (los tradicionales Filosofía para niños, ADAPT, DOORS, DORIS; o más actualizados como ADDIE, etc.) enmarcados en el enfoque propuesto como intrapersonal. Aquellos que promueven el uso contextualizado y cultural del pensamiento como habilidad para intervenir en la realidad social y con los otros (programas enfocados en resolución de problemas como CoRT, ASARPIC, ARDESOS; o aquellos centrados en el debate argumentativo y la escritura crítica) en el enfoque propuesto como interpersonal.

De todos modos creemos que hay mucho espacio aún para reflexionar y aportar en la comprensión de este tema que resulta llamativo para la formación profesional y la educación universitaria, y en especial, desde miradas innovadoras y complejas que brinden una perspectiva integradora del pensamiento crítico, capaz de incorporar las diferentes habilidades (cognitivas, motivacionales, discursivas, etc.) que han estado presentes en este constructo, y así mismo, considerar las dos grandes facetas del conocimiento (como proceso individual y como proceso social) en un modelo válido y aplicable. Es esto último el siguiente paso que creemos necesario, de manera que la psicología aporte una propuesta compleja en este tema.

\section{Referencias}

Abrami, P. C., Bernard, R. M., Borokhovski, E., Wade, A., Surkes, M. A., Tamim, R., \& Zhang, D. A. (2008). Instructional interventions affecting critical thinking skills and dispositions: A stage one metaanalysis. Review of Educational Research, 78(4), 1102-1134.

Akdere, N. (2012). Turkish pre-service teachers' critical thinking levels, attitudes and self-efficacy beliefs in teaching for critical thinking. Thesis submitted for the degree of doctor in philosophy in the Department of Educational Sciences. Graduate School of Social Sciences of Middle East Technical University, Singapore. Disponível:http://citeseerx.ist.psu.edu/viewdoc/download?doi=10. 1.1.633.5347\&rep=rep1\&type=pdf

Antequera, G. (2011). La promoción del pensamiento crítico en el aprendizaje basado en problemas (ABP). Un análisis a partir de los instrumentos de medición. Observar, 5, 68-94.

Beas, J., Santa Cruz, J., Thomsen, V., \& Utreras, S. (2003). Enseñar a pensar para aprender mejor. Santiago: Edic. Universidad Católica de Chile.

Beltrán, M. \& Torres, N (2009). Caracterización de habilidades de pensamiento crítico en estudiantes de Educación Media a través del test HCTAES. Zona Próxima, 11, 66-85.

Bensley, D.A. \& Spero, R. (2014). Improving critical thinking skills and metacognitive monitoring through direct infusion. Thinking Skills and Creativity, 12, 55-68.

Bouzas, P. G. (2009). Aprendizaje cooperativo. Papel del conflicto sociocognitivo en el desarrollo intelectual. Consecuencias 
pedagógicas. Revista española de Pedagogía, 131-148.

Butler, H. A. (2012). Halpern Critical Thinking Assessment Predicts Real-World Outcomes of Critical Thinking. Applied Cognitive Psychology, 26(5), 721-729.

Carmona, G. (2005). Investigación ética y educación moral: el Programa de Filosofía para Niños de Matthew Lipman. Revista de Artes y Humanidades UNICA, 6(12), 101-128.

Daniel, M. \& Auriac, E. (2011). Philosophy, Critical Thinking and Philosophy for Children. Educational Philosophy and Theory, 43(5). Doi: 10.1111/j.1469-5812.2008.00483.x

Davies, M. (2013). Critical thinking and the disciplines reconsidered. Higher Education Research \& Development, 32(4), 529 - 544. Doi: 10.1080/07294360.2012.697878

De Bono, E. (2009). Cort 1: Herramientas para ampliar el pensamiento. USA: The McQuaig Group Inc.

Dwyer, C., Hogan, M.J., \& Stewart, J. (2012). An evaluation of argument mapping as a method of enhancing critical thinking performance in e-learning environments. Metacognition Learning, 7, 219-244. Doi: 10.1007/s11409-012-9092-1

Eterovic, C. \& Stiepovich, J. (2010). Enfermería basada en la evidencia y formación profesional. Ciencia y enfermería, 16(3), 9-14.

Govier, T. (2010). A practical study of argument .Wadsworth, Cengage Learning: Belmont, CA.

Guba, E. \& Lincoln, Y. (2002). Paradigmas en competencia en la investigación cualitativa. In: Denman, C.; Haro, J. (Orgs.), Antología de métodos cualitativos en investigación social, 113-145. El colegio de Sonora: Sonora.

Harada, E. (2009). Algunas aclaraciones sobre el "modelo" argumentativo de Toulmin. Contactos, 73, 45-56.

Hawe, G. (2003). Pensamiento crítico en la formación universitaria. Documento de Trabajo 2003/6. Proyecto Mecesup TAL 0101. Recuperado: 11 mai. 2016. Disponível: http://www.freewebs.com/gustavohawes/Educacion\%20 Superior/2003\%20PensamientoCritico.pdf

Hernández, S.C. (2007). El constructivismo social como apoyo al aprendizaje en línea. Apertura impresa, 7(7), p46-62.

Kozulin, A. (2006). Individualismo epistemológico frente a una posición sociocultural: Piaget, Vigotsky y la teoría del aprendizaje mediado. In: D. Páez \& A. Blanco (Orgs.), La teoría sociocultural y la Psicología social actual. Madrid: Ed. Fundación Infancia y Aprendizaje.

Ku, K. \& Ho, I. (2010). Metacognitive strategies that enhance critical thinking. Metacognition Learning, 5, 251-267. Doi: 10.1007/ s11409-010-9060-6
López, G. (2012). Pensamiento crítico en el aula. Docencia e Investigación, 37(22), 41-60.

Marin, L. \& Halpern, D. (2011). Pedagogy for developing critical thinking in adolescents: Explicit instruction produces greatest gains. Thinking Skills and Creativity, 6, 1-13.

Marinkovich, J. (2007). Las estrategias cognitivo-retóricas y la dimensión dialéctica de la argumentación oral en una clase de lengua castellana y comunicación. Revista Signos, 40(63), 127146.

Marková, I. (2006). En busca de las dimensiones epistemológicas de las representaciones sociales. Em D. Páez \& A. Blanco (Orgs.), La teoría sociocultural y la Psicología social actual. Madrid: Ed. Fundación Infancia y Aprendizaje.

Martínez, M. \& Pascual, M. (2013). La influencia de la enseñanza virtual sobre el pensamiento crítico de los profesores en formación. Profesorado, revista de curriculum y formación del profesorado, 17(3), $293-306$.

Mateos, M. (2001). Metacognición y Educación. Bs. Aires: Aique.

Montero, M. (2010). Crítica, autocrítica y construcción de teoría en la psicología social latinoamericana. Revista colombiana de psicología, 19(2), 177-191.

Mota de Cabrera, C. (2010). Desarrollo del pensamiento crítico a través del discurso argumentativo: Una experiencia pedagógica en un curso de lectura y escritura. Entre Lenguas, 15, 11-23.

Nickerson, R., Perkins, D., \& Smith, E. (1998). Enseñar a Pensar. Madrid: Paidos.

Olivares, S. \& Heredia, Y. (2012). Desarrollo del pensamiento crítico en ambientes de aprendizaje basado en problemas en estudiantes de educación superior. Revista Mexicana de Investigación Educativa, 17(54), 759-778.

Olivares, S., Saiz, C., \& Rivas, S. (2013). Motivar para pensar críticamente. Electronic Journal of research in Educational Psychology, 11(2), 367-394.

Oyler, D. R. \& Romanelli, F. (2014). The Fact of Ignorance Revisiting the Socratic Method as a Tool for Teaching Critical Thinking. American Journal of Pharmaceutical Education, 78(7), 1-9. Doi: 10.5688/ajpe787144

Parra, E. \& Lago de Vergara, D. (2003). Didáctica para el desarrollo del pensamiento crítico en estudiantes Universitarios. Educación Médica Superior, 17(2).

Paul, R. \& Elder, L. (2003). La mini-guía para el Pensamiento crítico. Conceptos y herramientas. Ed. Fundación para el Pensamiento Crítico. Recuperado: 11 mai. 2016. Disponível: www.criticalthinking. org/resources/PDF/SP-ConceptsandTools.pdf 
Saiz, C. \& Rivas, S.F. (2008). Evaluación en pensamiento crítico: una propuesta para diferenciar formas de pensar. Ergo, Nueva Época, $22-66$

Saiz, C. \& Rivas, S.F. (2011). Evaluation of ARDESOS program an initiative to improve critical thinking skills. Journal of scholarschip of teaching and learning, 11(2), 34-51.

Sánchez, I. (2012). Evaluación de una Renovación Metodológica para un Aprendizaje Significativo de la Física. Formación Universitaria, 5(5), 51-65.

Sotolongo, P. \& Delgado, C. J. (2006). El pensamiento crítico ante la complejidad social. In: P. Sotolongo \& C.J. Delgado (Orgs.), La revolución contemporánea del saber y la complejidad social. Buenos Aires: CLACSO.

Tejada, J. (2000). La educación en el marco de una sociedad global: algunos principios y nuevas exigencias. Profesorado, revista de currículum y formación del profesorado, 4(1). Recuperado: 11 mai. 2016. Disponível: http://digibug.ugr.es/bitstream/10481/18983/1/ rev41ART1.pdf

Tiwari, A., Lai, P., So, M. \& Yuen, K. (2006). A comparision of effects of problem based learning and lecturing on the development of students' critical thinking. Medical Education, 40(6), 547-554.

Valenzuela, J., Nieto, A., \& Muñoz, C. (2014). Motivación y disposiciones: enfoques alternativos para explicar el desempeño de habilidades de pensamiento crítico. Revista electrónica de investigación educativa, 16(3), 16-32.

Yang, Y. T. (2012). Cultivating critical thinkers: Exploring transfer of learning from pre-service teacher training to classroom practice. Teaching and Teacher Education, 28, 1116 -1130.

Zamudio, B., Rolando, L., \& Ascione, A. (2002). Argumentación, pensamiento crítico y metacognición. Buenos Aires: Oficina de Publicaciones Ciclo Básico Común.

Recebido em: 03 de outubro de 2016 Aprovado em: 06 de abril de 2017

\section{Sobre os autores}

Carlos Javier Ossa Cornejo (cossa@ubiobio.cl)

Psicólogo, Doctorem Psicología,Académico Departamento de Ciencias de la Educación. Universidad delBio-Bio, Chillán, Chile.

Alejandro Díaz Mujica (adiazm@udec.cl)

Psicólogo, Doctor em Psicología, Académico Departamento de Psicología. Universidad de Concepción, Concepción, Chile.

Esta investigación se desarrolló con el apoyo del proyecto FONDECYT 1161502, "Modelo explicativo de la permanencia y el abandono de los estudios universitarios, basado en procesos cognitivo motivacionales", y CONICYT Chile (CONICYT-PCHA / Doctorado Nacional / 2015-21150389). 